\title{
Aqualabyrinth - A New Enrichment for Laboratory Zebrafish Under Analysis for Future Production
}

Dorothee Therrien ( $\nabla$ dorothee.therrien@muhc.mcgill.ca )

Research Institute of McGill University Health Center (RI-MUHC)

Aurore Dodelet-Devilliers

Research Institute of McGill University Health Center (RI-MUHC)

\section{Article}

Keywords: laboratory Zebrafish (Danio rerio), environmental enrichment, Aqualabyrinth

Posted Date: September 8th, 2021

DOI: https://doi.org/10.21203/rs.3.rs-845879/v1

License: (c) (i) This work is licensed under a Creative Commons Attribution 4.0 International License. Read Full License 


\section{Abstract}

In the field of laboratory animals, there have been considerable improvements in environmental enrichment for mammalian species, but little remains available for aquatic species, in particular fish. Recent publications have shown benefits in providing laboratory Zebrafish (Danio rerio) certain types of enrichments, such as substrate and plants. This research article proposes a novel tank enrichment called the Aqualabyrinth, and the benefit of its use in laboratory Zebrafish. While the enrichment won 3rd place at the 2020 Janet Wood Innovation Award (JWIA) competition, the Aqualabyrinth is not yet available on the market. This novel enrichment proposes similar if not more qualities than current aquatic enrichments commercially available. More specifically, its ease of usage, maintenance, and modular configuration options make it an ideal choice for Laboratory fish tanks. Results showed interaction on average up to 10.5 per 3 fish within a 30 second test interval versus 6.25 per 3 fish within 30 seconds interval for a mouse igloo. With the additional benefits highlighted in this study, the Aqualabyrinth proves to be an efficient and practical environmental enrichment option, pending collaboration with interested manufacturers.

\section{Introduction}

Aquatic species, in particular small zebrafish (Danio rerio) and medaka (Oryzias latipes) , are being used more and more as an animal model in science, for their small size, large transparent embryo and genetic similarities with human diseases [1]. As a result, there have been considerable improvements in environmental enrichment procedures for these non-aquatic species. However, like many other studies on aquatic species husbandry and care, there is still little knowledge on what exactly those animals need to increase their well-being in terms of enrichment. Previous evidence from studies done on single housed fish showed that social interaction was preferred to inanimate objects, resulting in lowering their anxiety and stress [2]. However, when cohousing is an option, a barren tank does not provide a shelter nor does it provide other sensory stimuli to the fish. In this situation, there is an increasing chance of stress due to dominance among the fish, injuries due to fighting, and possible boredom. Increased cortisol levels can in fact decrease growth rate, fertility, and egg count [3]. In addition, when different types of environmental enrichments were tested to see if there was preference and how the behavior of the fish in the tank changed, results have showed that shaded tanks, barren tanks, PVC material and overcrowding (too many plants) increase stress and/or aggressivity and plastic material (over PVC), shaded refuge, and submerged plant reduced aggressivity and/or cortisol and/or increase behavior diversity [3].

This report aims to pay some attention to aquatic species and to describe a new enrichment designed for small laboratory fish, such as the Zebrafish. The design has considered some of the problems common with many aquatic species, and a prototype was fitted to the Aquaneering Inc. tanks. The enrichment was made versatile so that it can be easily customized for other types of tank. The main goal of Aqualabyrinth product is to help mimic a natural aquatic environment and to provide the fish with a place to explore, hide, as well as a safe environment to be co-housed and to reduce stress, anxiety. The design is considering some important specifications that allow for ease of manufacturing and assembly and low 
cost of the product production. The DFMA technique (Design for Manufacturing and Assembly) was followed in an early design phase of the project in order to minimize product cost through design and process improvements. The Aqualabyrinth was put under experiment to better show its efficacy and to encourage laboratory enrichment companies to collaborate with the authors to seek manufacturing so that this novel enrichment can be accessible to the many aquatic facilities.

\section{Materials And Methods}

\section{Housing \& Husbandry}

The Zebrafish involved in the experience were of $A B$ strained originally supplied by a donating laboratory and housed in Aquaneering 2.8 and 6 liters tanks in reverse osmosis water supplied with balanced salt and bicarbonate solutions, heated at $27^{\circ}$ Celcius with light:dark cycle 10:14 exposure 10Am to 12AM. Water $\mathrm{pH}$, temperature, and conductivity is monitored daily, while nitrate, nitrites, hardness and alkalinity is monitored weekly, to ensure acceptable water quality. Fish are fed twice daily with Gemma Micro 300 (Skretting, USA). Fish were housed in groups of 3 fish or more. All experiments were performed under Institutional Animal Care and Use Committee-approved protocols at the Research Institute of McGill University Health Centre.

\section{Equipment}

\section{Material :}

$2 \times$ Vertical Large Panels (A) (Figure 1)

$2 \times$ Vertical Small Panels (B) (Figure 1)

2 x Horizontal Small Panels (C) (Figure 1)

$2 \times$ Red Igloos (Figure 2)

$5 \times 2.8$ liters Aquaneering Inc. Laboratory Fish Tanks

1 x 6 liters Aquaneering Inc. Laboratory Fish Tank

$1 \times$ Metal Tally Counter

\section{Testing Enrichments:}

(1) Vertical Large + Vertical Small Panels in a 2.8 liters tank

(2) Vertical Large + Horizontal Panels in a 2.8 liters

(3) Vertical Large + Horizontal Panels in a 6 liters tanks 
(4) Horizontal Panel in a 2.8 liters tank

(5) Igloo in a 2.8 liters tank

\section{Testing Aquariums \& Recording}

The components of the Aqualabyrinth were produced by the Janet Wood Innovation Award (JAWI) group as a prototype. The different panels consisted of a small vertical panel, a large vertical panel, and a small horizontal panel (Figure 1). As a comparison, we used mouse Igloos (Bioserv, NJ, USA). In each 2.8L tank except for Testing Tank (4), 3 adult fish were housed together (males and females mixed together). For Testing Tanks (3) and (4), 17 adult fish were together. For Testing Tanks (3) and (4), data were adapted such that we can compare with the other tanks. Namely, the approximate number of interactions from 17 to 3 fish was done by divinding the total number of interactions in 30 seconds by the number of fish (17 fish) and tehn multiplying this value by 3 fish.

The tanks were placed such that they all had neighbour tanks on the right and left side to rule out side preference during the experiment. The fish were provided 1 type of enrichment and were acclimated to it 5 days or more before the recording of the data.

To record and compare the different enrichment interactions, videos of 2.5 minutes were taken during a non feeding time of the day ( 1 hour after the first feeding of the day was performed) and the camera was set to record without human presence to avoid distracting the fish.

\section{Analysis}

A 30 seconds time frame was taken at 60 seconds and at 90 seconds and the average was calculated to analyse the behavior. For the igloo, the number of times a fish passed at a minimum distance of $1 \mathrm{~cm}$ of the enrichment and above or below was counted as an interaction and for the Aqualabyrinth configurations, the number of times a fish passed through an opening was counted as an interaction. Two tanks with enrichment (5) were used to collect data. For all the other enrichments (1)(2)(3)(4), 1 tank for each was set up to gather data.

\section{Results}

Table 1: Results of the Counts of each 30 Second Window 


\begin{tabular}{|c|c|c|}
\hline Testing Enrichment & $\begin{array}{c}\text { Counts during } 30 \\
\text { sec }\end{array}$ & $\begin{array}{l}\text { Average } \\
\text { Counts }\end{array}$ \\
\hline $\begin{array}{l}\text { (1) Vertical Large + Vertical Small Panels in a } 2.8 \\
\text { liters tank }\end{array}$ & $\begin{array}{l}\text { at } \mathrm{t}=60 \text { seconds: } \\
12 \\
\text { at } \mathrm{t}=90 \text { seconds: } \\
9\end{array}$ & 10.5 \\
\hline (2) Vertical Large + Horizontal Panels in a 2.8 liters & $\begin{array}{l}\text { at } t=60 \text { seconds: } \\
8 \\
\text { at } t=90 \text { seconds: } \\
4\end{array}$ & 6 \\
\hline $\begin{array}{l}\text { (3) Vertical Large + Horizontal Panels in a } 6 \text { liters } \\
\text { tanks }\end{array}$ & $\begin{array}{l}\text { at } t=60 \text { seconds: } \\
7 \\
\text { at } t=90 \text { seconds: } \\
6\end{array}$ & 6.5 \\
\hline (4) Horizontal Panel in a 2.8 liters tank & $\begin{array}{l}\text { at } t=60 \text { seconds: } \\
5 \\
\text { at } t=90 \text { seconds: } \\
7\end{array}$ & 6 \\
\hline (5) Igloo in a 2.8 liters tank & $\begin{array}{l}\text { tank } \# 1 \\
\text { at } \mathrm{t}=60 \text { seconds: } \\
8 \\
\text { at } \mathrm{t}=90 \text { seconds: } \\
5 \\
\text { tank } \# 2 \\
\text { at } \mathrm{t}=60 \text { seconds: } \\
7 \\
\text { at } \mathrm{t}=90 \text { seconds: } \\
5\end{array}$ & 6.25 \\
\hline
\end{tabular}

\section{Discussion}

In this study, both the mouse Igloo and the Aqualabyrinth were used as enrichment for Zebrafish. It is to be noted that interaction with the enrichments has been used here as a simple way to measure its use in fish. Mouse igloo interactions were not as clear as the ones for the Aqualabayrinth, and comparison between objects was made as close as possible. Both types of enrichment allowed similar interactions in similar conditions.

Both types of enrichment allowed proper monitoring of the health of the fish with their semi-transparent finish, but the cleaning of the igloo is more labour intensive due to the black rubber material and the dome-shaped. Indeed, the rubber may increase dirt and algae accumulation and the dome shape can be 
tricky to clean on the concave side. Aqualabyrinth design is flat and has only acrylic which makes it easy to clean and handle.

It is interesting to note the difference in terms of space and shape they take. Igloos do not allow multiple ways to use it (either up or upside down). Aqualabyrinth (even using a one panel solely) on its side can be very versatile and can fit in different angles and different sized tanks. As shown in the methodology section, with only 3 different types of panel, laboratory facilities can easily create at least 5 different configurations and with those configurations, the different size of tanks also gives different appearance outcomes and will influence how the fish will behave. Indeed, Aqualabyrinth could potentially prevent stereotypical circling behavior as the variety of configurations allows the fish to have a new experience as frequently as needed. It is important to note that depending on the fish habituation to the enrichment, the fish may interact differently. One tank was analyzed after the experiment with 3 fish and the horizontal panel in the $2.8 \mathrm{~L}$ tank and they were not utilizing the enrichment. Rather, they were staying at the top of the enrichment and chasing each other. This tank had males and females fish and were observed during a 2 day-period. A hypothesis could be that some configurations may induce egg laying behaviour as it may mimic shallow waters. Also, like for any other animals, when fish are housed without enrichment, once an adult, it may be more difficult to understand the concept of navigating through holes and using the enrichment. Indeed, it is seen that captive animals, versus wild animals, do not have the same basic hunting instinct [4]. That being said, early exposure to any type of enrichment is important so that the animals can be less stressed, develop more nerve connections making them more apt to interact with their environment, and to avoid stereotypical behaviors.

In addition to alleviating stereotypical behaviours, fighting could be seen to be diminished using the Aqualabyrinth. In the author's own personal home tank, Zebrafish were seen to stop fighting when using the Aqualabyrinth. To further experiment the potential effect on the fighting, longer studies should be performed.

The common arguments of lack of environmental enrichment options for aquatic species often revolve around impact of water quality, potential risk of disease spread due to the biofilm accumulation on the structures, and that diverse environmental enrichment may lead to experimental variation [3]. However, careful selection of the material used to put into the water or use of outside backgrounds will not affect water quality. Using proper tank cleaning as well as using good material for enrichment will reduce biofilm accumulation. Lastly, experimental variation is possible, yet in non-aquatic animals such as rats and mice, enrichments are mandatory unless justified and there are ways to make the study consistent by having the same enrichment for all tanks of the experiment. It is needless to say, happy animals make good science and this leads to quality scientific data. Namely, if enrichment does work, preventing stress and fight wounds, then the investment becomes worth it [2].

Upon reading this article, the author wishes that one could see the same potential as her and decide to manufacture the product with her collaboration so that it becomes readily available on the Laboratory Market. 


\section{Declarations}

Funding: This work received funding from DateSand Group.

Institutional review Board Statement: Not applicable.

\section{References}

[1] Choi TY, Choi TI, Lee YR, Choe SK, Kim CH. Zebrafish as an animal model for biomedical research. Exp Mol Med. 2021;53(3):310-317. doi:10.1038/s12276-021-00571-5

[2] Krueger. L. D, Thurston. S. E, Kirk. J, Elsaeidi. F, Freeman. Z. T, Goldman. D, Lofgren. J. L, Keller. J. M, "Enrichment Preferences of Singly Housed Zebrafish (Danio rerio)", Journal of the American Association for Laboratory Animal Science. vol 59, no 2, pp. 148-155, 2020

[3] Stevens. C. H, Reed. B. T, Hawkins. P, “Enrichment for Laboratory Zebrafish - A Review of the Evidence and the Challenges", MDPI, Animals, 2021, 11, 698. https://doi.org/10.3390/ani1103698

[4] Cormier. Z, "Can Captive Animals Ever Truly return to the Wild?", $B B C$ earth. https://www.bbcearth.com/news/can-captive-animals-ever-truly-return-to-the-wild

\section{Graph}

Graph 1 is available in the Supplementary Files section

\section{Figures}




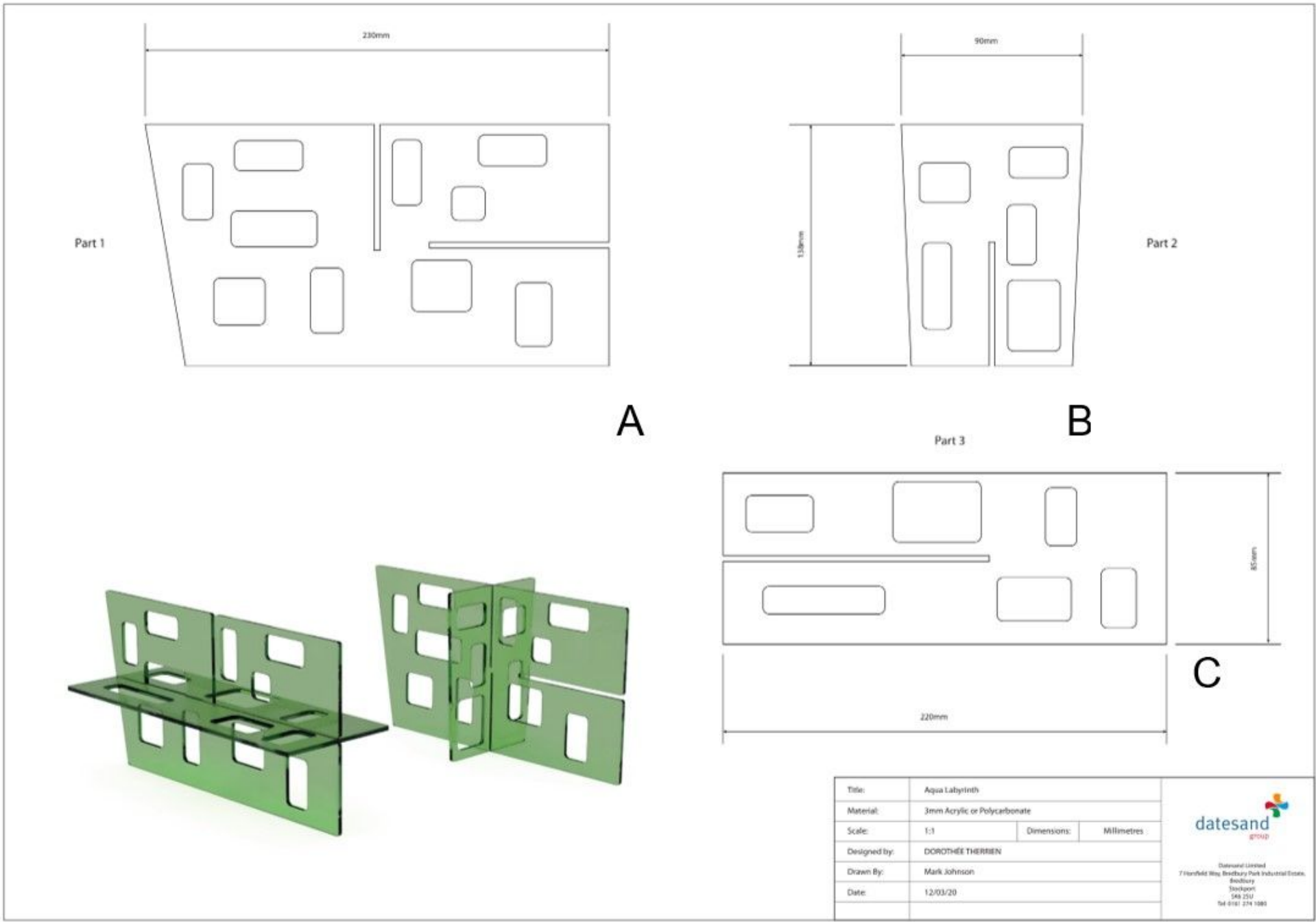

Figure 1

Technical Drawing of the Aqualabyrinth 


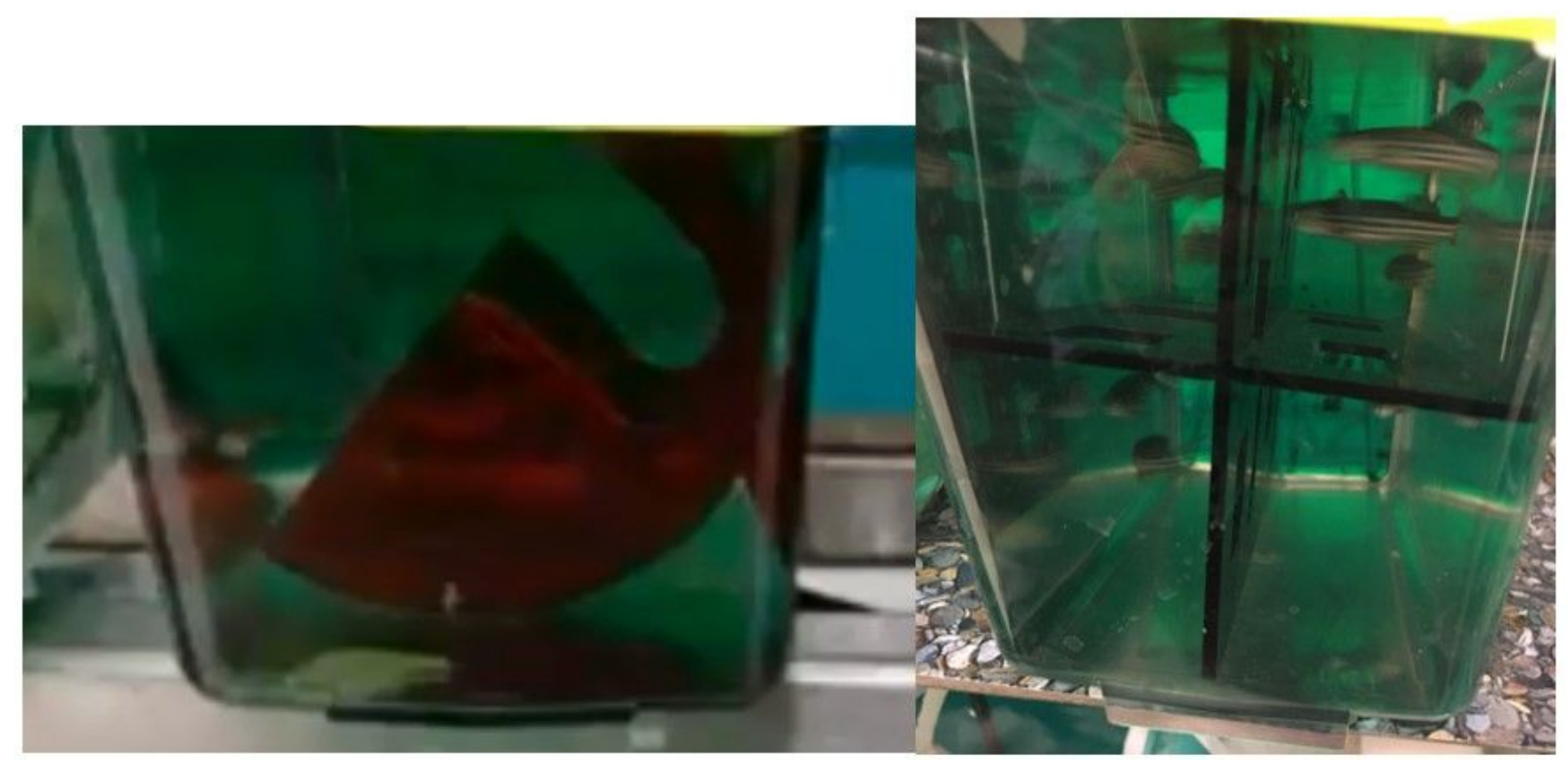

Figure 2

Mouse Igloo $(\mathrm{L})$ and Aqualabyrinth $(\mathrm{R})$

\section{Supplementary Files}

This is a list of supplementary files associated with this preprint. Click to download.

- Graph1.jpg

- Aqualab.jpg

- 110x802021DTFINAL.pptx.pdf 